\title{
Re: The Study of Energy Metabolism in Bladder Cancer Cells in Co-culture Conditions Using a Microfluidic Chip
}

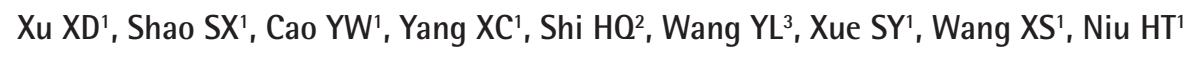 \\ ${ }^{1}$ The Affiliated Hospital of Qingdao University Medical College, Clinic of Urology, the Key Laboratory of Urology, Qingdao, China \\ ${ }^{2}$ Second Military Medical University, Shanghai Hospital, Clinic of Urology, Shanghai, China \\ ${ }^{3}$ Shanghai Jiao Tong University School of Medicine, Shanghai Institute of Andrology, Ren Ji Hospital, Department of Urology, Shanghai, China
}

Int J Clin Exp Med 2015;8:12327-12336.

\section{EDITORIAL COMMENT}

As we well know, the "Warburg effect" is the main theory of the energy metabolism in cancer cells. In contrast to normal differentiated cells, which rely primarily on mitochondrial oxidative phosphorylation to generate the energy needed for cellular processes, most cancer cells instead rely on aerobic glycolysis. This theory was described by Otto Warburg in 1924. In this research, the authors aimed to systematically analyze changes in mitochondrial-related protein expression in bladder cancer cells and tumor-associated fibroblasts and to investigate the characteristics of bladder cancer cell energy metabolism.

The authors reported that the energy metabolism of bladder tumor cells did not parallel the "Warburg effect" because even under sufficient oxygen conditions, cancer cells still underwent glycolysis. In this research, bladder cancer cells also had an efficient oxidative phosphorylation process wherein cancer cells promoted glycolysis in adjacent interstitial cells, thereby, causing increased formation of nutritional precursors. These high-energy metabolites were transferred to adjacent tumor cells in a specified direction and entered the Krebs cycle. As a result of this research, oxidative phosphorylation increased, and sufficient adenosine triphosphate was produced.

Therefore, in the near further, many studies on the energy metabolism of the urological cancer cell models will be pioneer to development of new target therapy options for cancer patient.

Fehmi Narter, MD, PhD

๑Copyright 2017 by the Association of Urological Surgery / Journal of Urological Surgery published by Galenos Publishing House. 